\title{
A simple method for the design of tension structures combining topological mapping and nonlinear structural analysis
}

\section{Un método sencillo de diseño de estructuras tensadas combinando mallado en topología y análisis no lineal de estructuras}

$\underline{\text { R. Jurado-Piña }}^{(*)}$, M. A. Salazar-Troya ${ }^{(* *)}$

\begin{abstract}
When designing a tension structure the shape is not known at the beginning of the process. Form-finding methods allow the designer to obtain an initial shape from given boundary conditions. Several form-finding methods for tension structures are already available in the technical literature; all of them posses certain limitations and drawbacks and no single method is optimal for all problems. The engineer may select the proper combination of methods best suited to the designer's needs. In this paper it is proposed a combined method to achieve satisfactory equilibrium configurations for fabric tension structures. The force density method (FDM) implemented with topological mapping (TM) is used as a search engine for the preliminary design, and a procedure that employs nonlinear structural analysis is proposed for final refinement of the initial equilibrium configuration hence allowing the use of the same analysis tool for both refinement of the solution and analysis under loading.
\end{abstract}

Keywords: Tension structures; form-finding; structural optimization.

\section{RESUMEN}

Al diseñar una estructura tensada la forma inicial es normalmente desconocida. Los métodos de búsqueda de forma permiten al ingeniero obtener una geometría inicial dadas unas condiciones de contorno. Existen diferentes métodos de búsqueda de formas de equilibrio, pero todos tienen limitaciones y no existe uno único óptimo para cualquier tipo de problema. El ingeniero debe elegir la combinación de métodos que mejor se adapte a sus necesidades. En este artículo se propone un método combinado para generar configuraciones de equilibrio satisfactorias en estructuras tensadas. Como motor de búsqueda para el diseño preliminar se emplea el método de las densidades de fuerza (FDM) implementado con mallado en topología (TM), y se propone un procedimiento basado en análisis no lineal de estructuras para el refinamiento de la configuración inicial de equilibrio, permitiéndose así el empleo de las mismas herramientas tanto para el refinamiento de la solución inicial como para el cálculo posterior bajo condiciones de carga.

Palabras claves: Estructuras tensadas; búsqueda de forma; optimización de estructuras.

(*) Technical University of Madrid. Spain.

(**) University of Granada. Spain.

Persona de contacto/Corresponding author: rafael.jurado@upm.es (R. Jurado-Piña)

Cómo citar este artículo/Citation: Jurado-Piña, R., Salazar-Troya, M. A. (2014). A simple method for the design of tension structures combining topological mapping and nonlinear structural analysis. Informes de la Construcción, 66(EXTRA-1): mo12, doi: http://dx.doi.org/10.3989/ic.13.090.

Licencia / License: Salvo indicación contraria, todos los contenidos de la edición electrónica de Informes de la Construcción se distribuyen bajo una licencia de uso y distribución Creative Commons Reconocimiento no Comercial 3.o. España (cc-by-nc). 


\section{INTRODUCTION}

The design of tension structures is a relatively complex problem because of the relationship between the stress distribution and the final shape (1) (2). The design of a tension structure (3) can be described in three steps: initial shapefinding, stress analysis of the structure and patterning. In the process of shape-finding (or form-finding), the designer gives a set of parameters, calculates other parameters and solves the shape of the structure that satisfies equilibrium. Under stress analysis, loads are applied to a structure whose initial equilibrium configuration is given by the previous step, and then the membrane adapts its configuration to the applied loads. Patterning is concerned with how a curved surface can be formed from fabric rolls; several techniques are available for this step.

Form-finding for tension structures is nowadays accomplished by numerical methods and computers. The parameters involved in the form-finding process are: i) type of element to model the fabric, ii) surface topology, iii) body forces, iv) surface tractions, v) nodal coordinates of the model, vi) geometry boundary conditions, and vii) internal stress distribution. Body forces and surface tractions are usually neglected during the shape-finding stage due to their insignificant influence in the geometrical shape at equilibrium. They can also complicate the initial equilibrium problem since the local direction and net magnitude of the loads may depend on an unknown initial surface shape. These loads are usually incorporated later into the stress analysis stage.

In most existing methods of equilibrium configuration, the shape is treated as an unknown. Then, it is necessary to introduce a set of parameters to ensure a unique solution. Ideally, one can use a nonlinear displacement analysis technique to solve the initial equilibrium problem using surface finite elements. In this case, the element stresses and the fixed nodes can be prescribed by the designer to guarantee a unique solution to the problem. The results of the equilibrium problem are the non-fixed nodes position. This technique has an important drawback: a geometrical initial mapping with a guess of the nodal coordinates is needed.

Several methods for solving the initial equilibrium problem (form-finding) have been developed to overcome some of the drawbacks associated with the nonlinear displacement method. In many of these methods a variety of limitations are imposed on the solution to transform the general nonlinear problem into a linear form. Siev and Eidelman (4) showed that if equilibrium is satisfied over a grid in the horizontal plane, vertical equilibrium can be used to calculate the elevation at the grid points. In the force density method (FDM) the membrane is modelled by a cable network. FDM is based upon the force-length ratios or force densities which are defined for each branch of the net structure; a simple example will be shown later. Schek (5) shows that the force densities are suitable for the description of the equilibrium state of any general network. The FDM renders a simple linear system of equations for a possible initial configuration.

Haber and Abel (6) presented the 'smoothing concept' which allows the designer to solve the shape of a reference configuration in terms of an assumed prestress distribution. Using this method the equilibrium equations are nonlinear and consequently iterative techniques are required for the solution.
Other methods of form-finding are based on dynamic analysis. One of the main contributors to these methods was Barnes (7) who used a method of dynamic relaxation with kinetic damping for form-finding.

Hernandez-Montes et al. (8) employed an important property of the FDM by which knowledge of the initial position of the non-fixed nodes of the structure is not needed; only the connectivity of the nodes is necessary to solve the problem. Accordingly, a new mapping method based on topology called topological mapping (TM) was presented; in contrast to the mapping methods used so far based exclusively on geometry.

Each of the methods mentioned above possesses certain limitations and drawbacks, therefore, no single solution method is optimal for all problems. The engineer may select the proper combination of methods best suited to the problem at hand and the designer's needs. In this paper we take advantage of some of the existing methods to propose a combined method for computing satisfactory equilibrium configurations. The objective is to develop a straightforward methodology to achieve final equilibrium configurations for fabric tension structures, making use of stress control over surface elements, and taking advantage of the FDM with topological mapping as preliminary shape-finding.

\section{DESCRIPTION OF THE PROCEDURE}

The procedure presented in this paper is shown in Figure 1. There are three stages with one or several steps. The first stage of the method uses topological mapping and the force density method (TM-FDM); in the second stage the obtained cable network is transformed into a membrane model; and in the third stage a new surface with a prescribed stress field is

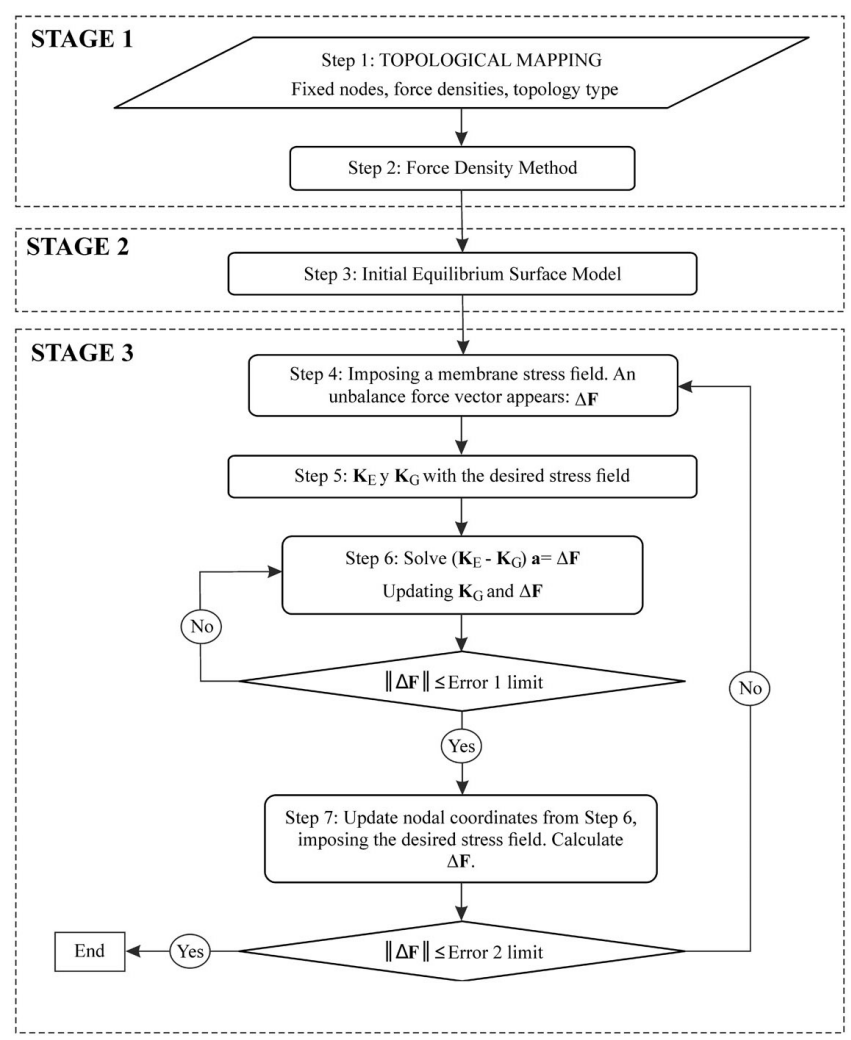

Figure 1. Flowchart of the applied method. 
searched. The details of the steps belonging to each stage are explained later along the manuscript.

In order to make the paper self-contained an introduction to FDM and TM are included.

\subsection{Initial equilibrium configuration}

\section{FORCE DENSITY METHOD}

The starting point for the FDM (5) is a pin-joint network consisting of cable or bar elements, in which some of the points are fixed and the others are free. The free points will have to find a position in the equilibrium configuration.

For a given pin-joint network with $\mathrm{n}$ nodes and $\mathrm{m}$ branches, the branch-node matrix $\mathrm{C}$ is a $m \times n$ matrix used in the FDM to define the connectivity of the nodes. Defining $i(j)$ and $k(j)$ as node $i$ and $k$ of branch $j$ respectively, for $i<k$ the elements of the branch-node matrix $\mathrm{C}$ can be built as follows (see Figure 2):

$$
\mathrm{C}(j, r)=\left\{\begin{array}{cc}
+1 & \text { if } i(j)=r \\
-1 & \text { if } k(j)=r \\
0 & \text { for the rest }
\end{array}\right.
$$

The nodes Pi have coordinates $\left(x_{i}, y_{i}, z_{i}\right), i=1, \ldots, \mathrm{n}$. Some of these nodes are fixed, and they will constitute the input data for the initial equilibrium configuration problem. If nodal forces with components $p_{i x}, p_{i y}$ and $p_{i z}$ are applied at each node $i$, letting $l_{j}$ and $s_{j}$ be the length and the branch force of each branch $j$, the equilibrium equations for each node may be established. In the case of Figure 2, the equilibrium equations for node 3 result from the projections of the forces along each axis:

$$
\begin{aligned}
& \frac{s_{1}}{l_{1}}\left(x_{3}-x_{1}\right)+\frac{s_{2}}{l_{2}}\left(x_{3}-x_{4}\right)+\frac{s_{3}}{l_{3}}\left(x_{3}-x_{2}\right)=p_{3 x} \\
& \frac{s_{1}}{l_{1}}\left(y_{3}-y_{1}\right)+\frac{s_{2}}{l_{2}}\left(y_{3}-y_{4}\right)+\frac{s_{3}}{l_{3}}\left(y_{3}-y_{2}\right)=p_{3 y} \\
& \frac{s_{1}}{l_{1}}\left(z_{3}-z_{1}\right)+\frac{s_{2}}{l_{2}}\left(z_{3}-z_{4}\right)+\frac{s_{3}}{l_{3}}\left(z_{3}-z_{2}\right)=p_{3 z}
\end{aligned}
$$

The great advantage of the FDM is the introduction of a parameter $q_{j}$, which represents the force-length ratio $\left(s_{j} / l_{j}\right)$ or force density for the branches. If $q_{j}$ is constant for all the branches then the equilibrium equations become a linear system.

Next, the $x, y$ and $z$-coordinates for each of the nodes are grouped in the $n$-vectors $x, y$ and $z$, and the nodal loads are characterized by means of $n$-vectors containing the force
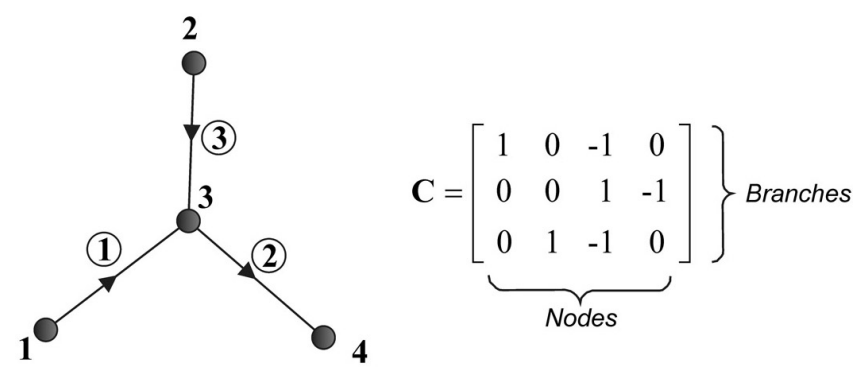

Figure 2. Example for the construction of the branch-node matrix. components $p_{x}, p_{y}$ and $p_{z}$. This allows to obtain a general formulation of the FDM for a complete network:

$$
\begin{aligned}
& \left(C^{T} Q C\right) x+p_{x}=0 \\
& \left(C^{T} Q C\right) y+p_{y}=0 \\
& \left(C^{T} Q C\right) z+p_{z}=o
\end{aligned}
$$

where $Q$ is the diagonal matrix that contains the force densities for the branches $q_{j}$. The above system of equations constitutes a linear system where the known values are the coordinates of the fixed points, the topology of the pin-joint network, and the force density values. The unknowns are the coordinates of non-fixed nodes. The $\mathrm{C}^{\mathrm{T}} \mathrm{QC}$ matrix is positive definite if we have a prestressed network $\left(q_{j}>0\right)$ without isolated points, so in this case we obtain exactly one equilibrium state by solving [3].

\section{TOPOLOGICAL MAPPING}

An initial guess of the shape is needed in order to generate a network, for instance the approximate shape of the horizontal projection. With this horizontal projection it is easy to consider a network even when only the connections are picked as input for the FDM; this way of doing is called "geometrical mapping", in order to differentiate it from the topological mapping method (TM).

The main feature of the topological mapping (TM) introduced by Hernández-Montes et al. (8) is that with a few topological rules a meshing can be performed independently of the final geometric configuration. TM considers two types of networks: closed and open networks. In this paper only closed networks will be used, so all explanations will be referred to this type of network.

The topology of the network is obtained with the following input data: the number of nodes in the second step, the number of steps and the type of relation between one step and the following one.

Three types of basic relations are defined between steps: A, B and C, see Figure 3. Relation A corresponds to a pattern in which each node at a given step is connected to the adjacent ones on the same step as well as three more nodes of the following step (Figure 3a). In relation $\mathrm{B}$, each node at a given step is connected to the adjacent ones on the same step as well as two nodes of the following step (Figure $3 \mathrm{~b}$ ). Case C is such that each node at a given step is connected alternatively to one or to three nodes of the next step, and also to the adjacent ones of the same step (Figure $3 \mathrm{c}$ ).

In order to correctly close the topological mapping a classification of nodes and connections has been defined for these networks. For the basic relationships A, B and C (Figure 3) there are three black nodes and one grey node in the second step. The grey node must coincide with the black one located on the border of the step, so the grey one is called 'repeated node'. Due to this procedure thick lines in Figure 3 are connections and dashed lines represent 'repeated connections'.

The construction of the network begins with one node which is connected to the n nodes of the second step. However, this initial node may be suppressed whenever there is the need 
to create a new interior contour in the net. Moreover, the nodes and ring branches located in the last step constitute the contour of the equilibrium shape. The number of nodes on each step is double the amount on the previous one when relationship type A is used, and remains constant when using a relationship type $\mathrm{B}$ or $\mathrm{C}$.

TM-FDM method assigns the fixed points to the nodes located at the last step through the calculation of the distance between consecutive fixed points and the perimeter formed by them in such a way that the distribution of nodes of the last ring is done proportionally to the real distance between the fixed points. This assignation is then performed automatically.

Figure 4a shows an example of tension structure meshed in topology with a sequence of basic relations A and B, and Figure $4 \mathrm{~b}$ shows another one with basic relation type $\mathrm{C}$ and with the consideration of an interior hole.

\subsection{Types of topological relations in TM-FDM}

The number of steps used in TM depends on the desired degree of refinement. A large number of steps will result in a mesh formed by a higher density of triangles. Once the number of steps has been decided, it is desirable to automatically generate the topological relations. These topological relations can be analyzed so as to achieve one goal: to obtain a mesh as uniform as possible, i.e., a mesh having triangles whose areas are as similar as possible. We have conducted an empirical analysis in which, for a given shape of the contour and a certain topological relationship, we have calculated the deviation from the average of the area of the triangles forming the equilibrium configuration. This analysis was repeated for a sample consisting of:

- Varying number of steps from 10-16.

- All possible topological relationships (once the number of steps is fixed).

- Different forms of the contour: regular and irregular.

From the obtained results, we have deduced a series of topological relations for the different number of steps. These relations are recommended in the generation of equilibrium configurations with the TM-FDM method and are shown in Table 1. Its use provides uniform meshes in the reached equilibrium configurations for both regular and irregular contour shapes.

\subsection{Transforming the cable model into a membrane model}

The constant stress triangular element is used; it is described in Figure 5 and 6. In the standard formulation of this element, the node equilibrium equation appears in the local coordinate system as a)

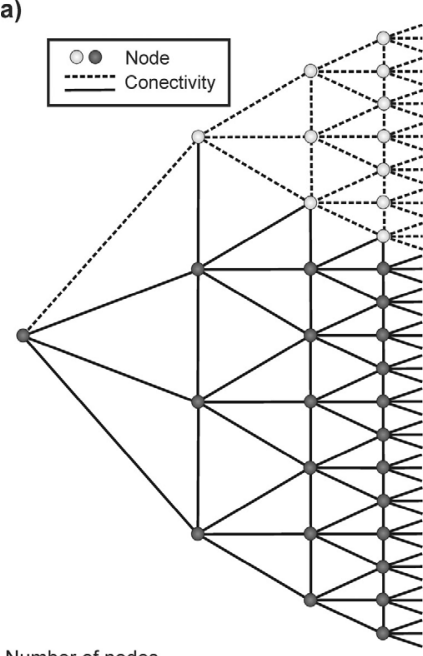

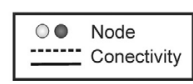

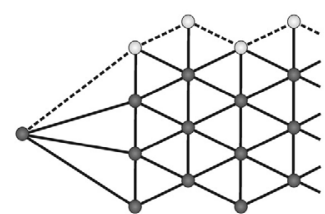

c)
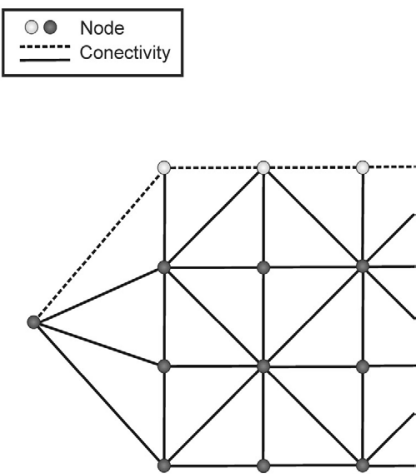

Number of nodes

Closed network: $n(3) \quad 2 n \quad 4 n \quad$ Closed network: $n(3) \quad n \quad n \quad n \quad \begin{array}{cllll}\text { Closed network: } & n(3) & n & n\end{array}$
Figure 3. a) Topology of network Type A. b) Topology of network Type B. c) Topology of network Type C

a)

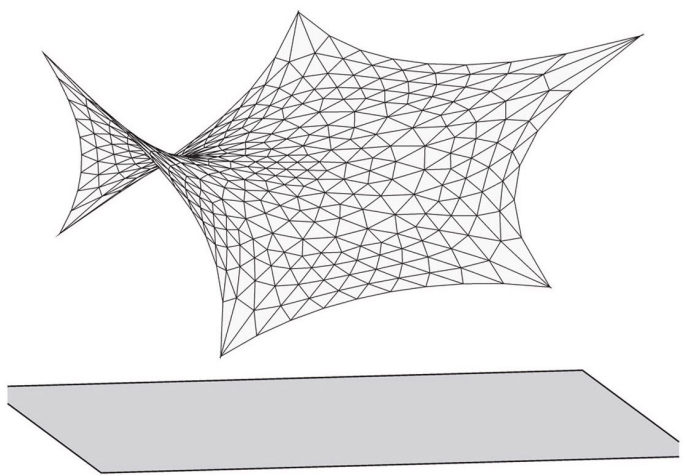

b)

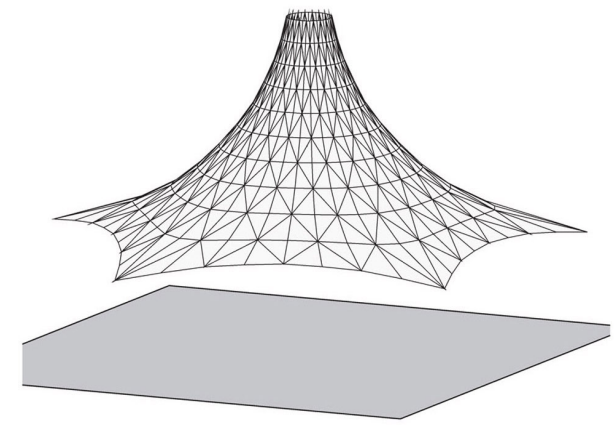

Figure 4. Examples of the use of meshing in topology a) using relations A and B b) using relation C and an interior hole. 
$\mathbf{f}^{\mathbf{e}}=\left(\begin{array}{c}\left(f_{i}^{e}\right)_{x} \\ \left(f_{i}^{e}\right)_{y} \\ \left(f_{j}^{e}\right)_{x} \\ \left(f_{j}^{e}\right)_{y} \\ \left(f_{m}^{e}\right)_{x} \\ \left(f_{m}^{e}\right)_{y}\end{array}\right]=\frac{t}{2} \cdot\left[\begin{array}{ccc}b_{i} & 0 & c_{i} \\ 0 & c_{i} & b_{i} \\ b_{j} & 0 & c_{j} \\ 0 & c_{j} & b_{j} \\ b_{m} & 0 & c_{m} \\ 0 & c_{m} & b_{m}\end{array}\right]\left(\begin{array}{c}\sigma_{x} \\ \sigma_{y} \\ \tau_{x y}\end{array}\right]=\frac{t}{2} \cdot\left[\begin{array}{c}b_{i} \sigma_{x}+c_{i} \tau_{x y} \\ c_{i} \sigma_{y}+b_{i} \tau_{x y} \\ b_{j} \sigma_{x}+c_{j} \tau_{x y} \\ c_{j} \sigma_{y}+b_{j} \tau_{x y} \\ b_{m} \sigma_{x}+c_{m} \tau_{x y} \\ c_{m} \sigma_{y}+b_{m} \tau_{x y}\end{array}\right]$

where $\sigma_{x}, \sigma_{y}, \tau_{x y}$ are the usual stresses of plane elasticity, $\mathrm{t}$ is the thickness of the membrane, fe is a vector that contains the nodal forces of the element, and b's and c's are defined as follow:

$$
\begin{aligned}
& b_{i}=y_{j}-y_{m} ; c_{i}=x_{m}-x_{j} \\
& b_{j}=y_{m}-y_{i} ; c_{j}=x_{i}-x_{m} \\
& b_{m}=y_{i}-y_{j} ; c_{m}=x_{j}-x_{i}
\end{aligned}
$$

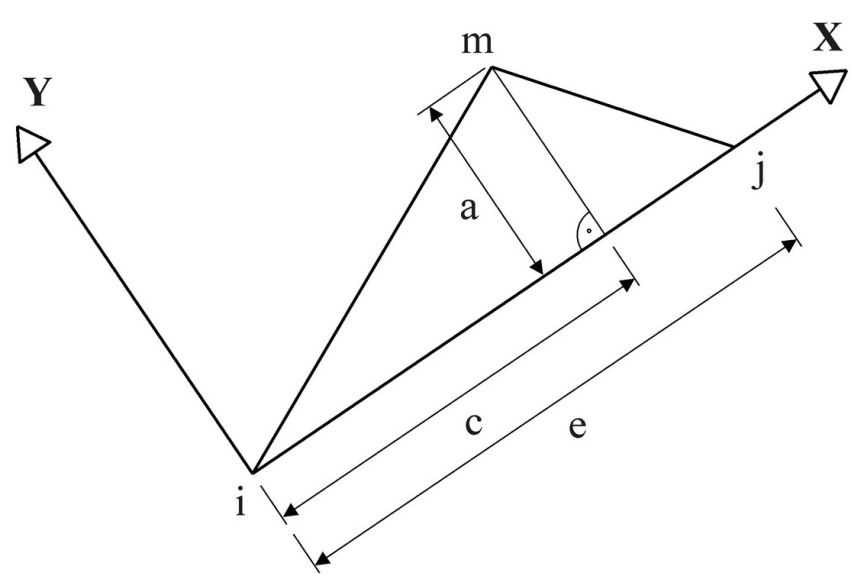

Figure 5. Triangular Finite Element in its Local Coordinate System.

A link is established between the stresses in a membrane model and the cable forces in its corresponding virtual triangulated network. First, a local coordinate system coplanar with the element is used. Afterwards, the components of the nodal forces (in a Cartesian system) are transformed into a skew system parallel to the edges of the element (Figure 6b), such that the following three independent equations can be obtained:

$$
\left(\begin{array}{c}
q_{i j} \\
q_{j m} \\
q_{m i}
\end{array}\right)=-\frac{t}{4}\left[\begin{array}{ccc}
b_{i} b_{j} & c_{i} c_{j} & b_{i} c_{j}+b_{j} c_{i} \\
b_{j} b_{m} & c_{j} c_{m} & b_{j} c_{m}+b_{m} c_{j} \\
b_{m} b_{i} & c_{m} c_{i} & b_{m} c_{i}+b_{i} c_{m}
\end{array}\right]\left(\begin{array}{c}
\sigma_{x} \\
\sigma_{y} \\
\tau_{x y}
\end{array}\right) \quad \text { [5] }
$$

where $\Delta$ is the area of the triangle. Let $Q_{r s}$ and $L_{r s}$ be the force and the length of the branch connecting the nodes $r$ and $s$ (Figure 6b). [5] renders the three force densities $\left(q_{r s}=Q_{r s}\right.$ $\left(L_{r s}\right)$ for a constant stress triangular element. For a given stress distribution over a membrane divided into triangles, [5] is used to obtain the three force densities for the virtual cable network in each triangle. In the case of an equilibrated network calculated by the force density method with topological mapping, the corresponding stress distribution over an equivalent finite element membrane model can be obtained as the inverse problem of [5]. This equilibrated membrane model will be referred as Initial Equilibrium Surface Model (IESM).

a)

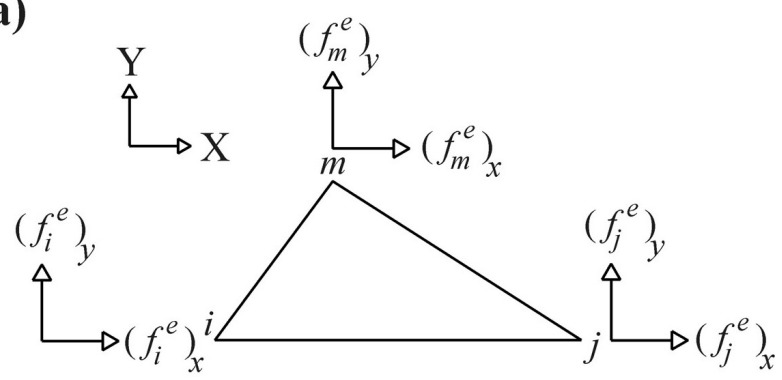

b)

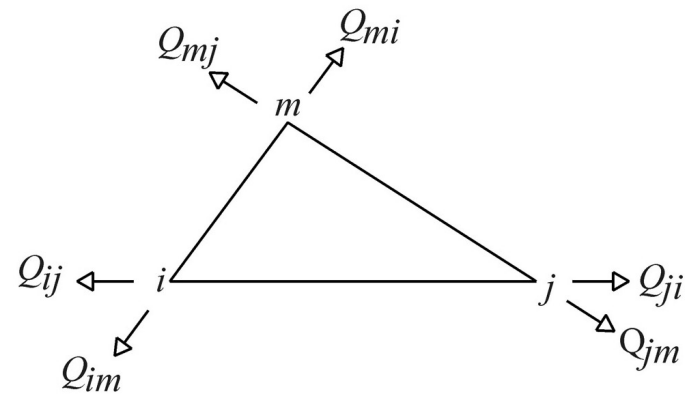

Figure 6. a) Standard constant stress triangular element

\begin{tabular}{|c|c|c|c|c|c|c|c|c|c|c|c|c|c|c|c|}
\hline \multirow{2}{*}{$\begin{array}{l}\text { NUMBER } \\
\text { OF STEPS }\end{array}$} & \multirow{2}{*}{$\begin{array}{l}\text { Number of nodes } \\
\text { for the second step }\end{array}$} & \multicolumn{14}{|c|}{ Topological relations from steps 3 to end } \\
\hline & & 3 & 4 & 5 & 6 & 7 & 8 & 9 & 10 & $\mathbf{1 1}$ & 12 & 13 & 14 & 15 & 16 \\
\hline 10 & 6 & A & $\mathrm{B}$ & A & $\mathrm{B}$ & $\mathrm{B}$ & A & $\mathrm{B}$ & $\mathrm{B}$ & & & & & & \\
\hline 11 & 6 & A & B & A & $\mathrm{B}$ & B & A & B & $\mathrm{B}$ & $\mathrm{B}$ & & & & & \\
\hline 12 & 6 & A & B & A & B & B & A & B & B & B & B & & & & \\
\hline \multirow{2}{*}{13} & 6 & A & $\mathrm{B}$ & A & $\mathrm{B}$ & $\mathrm{B}$ & $\mathrm{A}$ & B & $\mathrm{B}$ & B & B & $\mathrm{B}$ & & & \\
\hline & 6 & A & $\mathrm{A}$ & B & B & $\mathrm{A}$ & B & B & B & B & $\mathrm{A}$ & B & & & \\
\hline \multirow{2}{*}{14} & 6 & $\mathrm{~A}$ & B & A & B & B & $\mathrm{A}$ & B & $\mathrm{B}$ & B & B & B & $\mathrm{B}$ & & \\
\hline & 6 & A & $\mathrm{A}$ & B & $\mathrm{B}$ & $\mathrm{A}$ & B & B & $\mathrm{B}$ & B & $\mathrm{A}$ & B & $\mathrm{B}$ & & \\
\hline \multirow{2}{*}{15} & 6 & A & $\mathrm{B}$ & A & B & B & $\mathrm{A}$ & B & B & B & B & B & $\mathrm{B}$ & B & \\
\hline & 6 & A & $\mathrm{A}$ & B & B & $\mathrm{A}$ & B & B & B & B & $\mathrm{A}$ & B & $\mathrm{B}$ & B & \\
\hline \multirow{2}{*}{16} & 6 & A & B & $\mathrm{A}$ & B & B & $\mathrm{A}$ & B & B & B & B & B & $\mathrm{B}$ & B & B \\
\hline & 6 & A & A & B & B & $\mathrm{A}$ & B & B & B & B & A & B & $\mathrm{B}$ & B & B \\
\hline
\end{tabular}
b) same element with equivalent nodal force components in the direction of the edges.

Table 1. Recommended topological relations based on the number of steps. 


\subsection{Final equilibrium configuration with stress control}

The shape of tension structures depends on their internal stress pattern. Nonlinear structural analysis is needed to obtain a new equilibrium configuration when loading (or a perturbation) is added to the Initial Equilibrium Surface Model. In this paper nonlinear analysis is employed with the hypothesis of large rotations and small strains, together with the constant stress triangular finite element previously described. New equilibrium configurations are obtained due to perturbations introduced in the system. Perturbations come from changes in the stress field, these changes generate non equilibrated nodal loads. New equilibrium configurations with subsequent geometric changes are obtained using the Stiffness Matrix Method (9). Following we briefly describe the stiffness matrix that we have employed for the constant stress triangular element.

\section{THE ELASTIC AND GEOMETRIC STIFFNESS MATRIX}

In case of the plane stress triangular finite element, the elastic stiffness matrix of the element $\left(\mathrm{K}_{\mathrm{E}}^{\mathrm{e}}\right)$ is (9):

$$
\mathbf{K}_{E}^{e}=\left[\begin{array}{ccc}
\left(\mathbf{K}_{E}^{e}\right)_{i i} & \left(\mathbf{K}_{E}^{e}\right)_{i j} & \left(\mathbf{K}_{E}^{e}\right)_{i m} \\
\left(\mathbf{K}_{E}^{e}\right)_{j i} & \left(\mathrm{~K}_{E}^{e}\right)_{j j} & \left(\mathbf{K}_{E}^{e}\right)_{j m} \\
\left(\mathbf{K}_{E}^{e}\right)_{m i} & \left(\mathbf{K}_{E}^{e}\right)_{m j} & \left(\mathbf{K}_{E}^{e}\right)_{m m}
\end{array}\right]
$$

The components of the previous matrix are:

$$
\left(K_{E}^{e}\right)_{r s}=\frac{t}{4} \cdot \frac{E}{1-v^{2}} \cdot\left[\begin{array}{ccc}
b_{r} b_{s}+\frac{1-v}{2} c_{r} c_{s} & v b_{r} c_{s}+\frac{1-v}{2} c_{r} b_{s} & 0 \\
v_{r} b_{s}+\frac{1-v}{2} b_{r} c_{s} & c_{r} c_{s}+\frac{1-v}{2} b_{r} b_{s} & 0 \\
0 & 0 & 0
\end{array}\right] \begin{array}{r}
r=i, j, m . \\
s=i, j, m .
\end{array}
$$

where E and $v$ are Young's modulus and Poisson's ratio. $\left(\mathrm{K}_{\mathrm{E}}^{\mathrm{e}}\right)$ $\mathrm{ij}$ is the element in row $\mathrm{i}$ and column $\mathrm{j}$ of the matrix $\mathrm{K}_{\mathrm{E}}^{\mathrm{e}}$.

The geometric stiffness matrix associated with the elastic stiffness matrix defined in [6] can be obtained by perturbation methods. The resulting geometric stiffness matrix $\left(\mathrm{K}_{\mathrm{G}}^{\mathrm{e}}\right)$ is (9):

$$
\mathbf{K}_{\mathrm{G}}^{e}=\frac{\mathrm{t}}{2} \cdot\left[\begin{array}{ccccccccc}
0 & 0 & 0 & -\tau_{x y} & \sigma_{x} & 0 & \tau_{x y} & -\sigma_{x} & 0 \\
0 & 0 & 0 & -\sigma_{y} & \tau_{x y} & 0 & \sigma_{y} & -\tau_{x y} & 0 \\
0 & 0 & \alpha_{\mathrm{i}} & 0 & 0 & \beta_{\mathrm{i}} & 0 & 0 & \lambda_{\mathrm{i}} \\
\tau_{x y} & -\sigma_{x} & 0 & 0 & 0 & 0 & -\tau_{x y} & \sigma_{x} & 0 \\
\sigma_{y} & -\tau_{x y} & 0 & 0 & 0 & 0 & -\sigma_{y} & \tau_{x y} & 0 \\
0 & 0 & \alpha_{\mathrm{j}} & 0 & 0 & \beta_{\mathrm{j}} & 0 & 0 & \lambda_{\mathrm{j}} \\
-\tau_{x y} & \sigma_{x} & 0 & \tau_{x y} & -\sigma_{x} & 0 & 0 & 0 & 0 \\
-\sigma_{y} & \tau_{x y} & 0 & \sigma_{y} & -\tau_{x y} & 0 & 0 & 0 & 0 \\
0 & 0 & \alpha_{\mathrm{m}} & 0 & 0 & \beta_{\mathrm{m}} & 0 & 0 & \lambda_{\mathrm{m}}
\end{array}\right]
$$

where

$$
\alpha_{r}=-\frac{e-c}{a e}\left(\mathrm{f}_{r}^{e}\right)_{y}-\frac{1}{e}\left(\mathrm{f}_{r}^{e}\right)_{x}
$$

$\beta_{r}=-\frac{c}{a e}\left(\mathrm{f}_{r}^{e}\right)_{y}+\frac{1}{e}\left(\mathrm{f}_{r}^{e}\right)_{x}$

$\lambda_{r}=\frac{1}{a}\left(\mathrm{f}_{r}^{e}\right)_{y}$

$r=i, j, m$

$a, e$ and $c$ are illustrated in Figure 5 .

\section{CONFIGURATIONS WITH STRESS CONTROL}

Given an initial equilibrium configuration, usually a different equilibrium shape close to the initial shape might be obtained, changing both the geometry and the stress distribution of the initial structure. For example, it may be attractive to obtain an equilibrium configuration with a constant stress field, or other prescribed stress patterns, by changing the Initial Equilibrium Surface Model (derived from the initial cable model equilibrium configuration obtained by FDM and TM). It is possible to generate equilibrium configurations with an imposed stress distribution using the Stiffness Matrix Method. The procedure is based on the same displacement analysis techniques used to study the behaviour of tension structures under varying load condition. In this method geometrical nonlinear analysis techniques are used to compensate unbalanced forces. These unbalanced forces result from the imposition of a new stress field to the Initial Equilibrium Surface Model. The applied procedure contains the following steps, schematically described in Figure 1:

- Step 1: Introduce the necessary data for the topology mapping: the coordinates of the fixed nodes, the topology of the pin-joint network and the force density values.

- Step 2. Calculate an initial equilibrium configuration based on the force density method. The coordinates of the nonfixed nodes are obtained.

- Step 3: Compute the membrane stresses using [5]. The Initial Equilibrium Surface Model is obtained.

- Step 4: Modify the membrane stresses to the desired values. This introduces a perturbation in the system, and an unbalanced force vector $(\Delta \mathrm{F})$ appears.

- Step 5: Assemble individual element stiffness matrices to form the elastic stiffness matrix $\mathrm{K}_{\mathrm{E}}$ and the geometrical stiffness matrix $\mathrm{K}_{\mathrm{G}}$ of the structure with the desired stresses.

- Step 6: Equilibrium requires that the sum of all forces must be zero at each node, so a new equilibrium configuration of the structure is obtained by allowing the deformation of the structure under the unbalanced forces $(\Delta \mathrm{F})$. [9] is solved by iterations, as a regular second order analysis:

$$
\left(\mathbf{K}_{\mathbf{E}}+\mathbf{K}_{\mathrm{G}}\right) \mathbf{a}=\Delta \mathbf{F}
$$

where a is the vector of nodal displacements and $\Delta \mathrm{F}$ is the vector of nodal forces resulting from the perturbation induced by the imposed stress field, which induces a non-equilibrium configuration. Each iteration requires the calculation of:

- The new nodal positions, adding to the previous nodal position the nodal displacements a.

- The vector of unbalanced forces $\Delta \mathrm{F}$, calculated with the stress field variation. Small variations are preferred for numerical convergence; the magnitude of these variations can be easily controlled with small values of the Young's modulus E.

- The error, which can be computed as the second norm of the vector $\Delta \mathrm{F}$ (I have named it as error Type 1). 
- In case the error (Type 1) is bigger than an upper bound value, $\mathrm{K}_{\mathrm{E}}$ and $\mathrm{K}_{\mathrm{G}}$ are recalculated taking into account the new nodal position and the new stress field.

- Step 7: Compute the vector of unbalanced forces $\Delta \mathrm{F}$ with the final nodal position obtained in Step 6 and the desired stresses. Finally, compute the error as the second norm of the vector $\Delta \mathrm{F}$ (Error Type 2). If this error is bigger than an upper bound value, go to Step 4, with the previous obtained nodal coordinates.

\section{EXAMPLE}

\section{Steps 1 and 2: Initial equilibrium configuration}

A tension structure with 8 fixed points is designed. The first step is the solution of the form-finding problem, TM and FDM is used. The necessary input data for this first step are the following:

- Boundary conditions:

Number of fixed points: 8

Coordinates of the fixed points: $(-6,-45,0),(6,-45,0)$, $(27,-24,15),(27,24,15),(6,45,0),(-6,45,0),(-27,24,15)$, $(-27,-24,15)$

- Topology:

Desired number of steps: 12

Type of network: obtained from Table 1 for the desired number of steps.
- Force density values

For the inner branches: 1

For the external branches: 10

With these simple inputs the geometry corresponding to the initial equilibrium configuration is automatically obtained (Figure 7).

\section{Step 3: Transforming the cable model into a membrane model}

The cable model is transformed into a membrane model composed by triangular elements; the stress (units of force per length) field is obtained by [5]. The corresponding stress distribution is shown in Figure 8. Stresses have been scaled so that the minimum value of the major principal stress is equal to 1 . At the exterior cables the obtained values vary between 59 and 34 with a mean value of 48 units of force.

\section{Steps 4 to 7. Final equilibrium configuration with stress control}

For simplicity, in this example an isotropic and uniform stress field has been chosen in the membrane. The fixed value of the principal stresses is 1 (force/length units). An imposed value of force of 48 (force units) has been given to the exterior cable. A Poisson's ratio equal to zero and a Young's modulus equal to 0.5 have been used. After few iterations (NewtonRaphson) from steps 4 to 7 a new equilibrium configuration

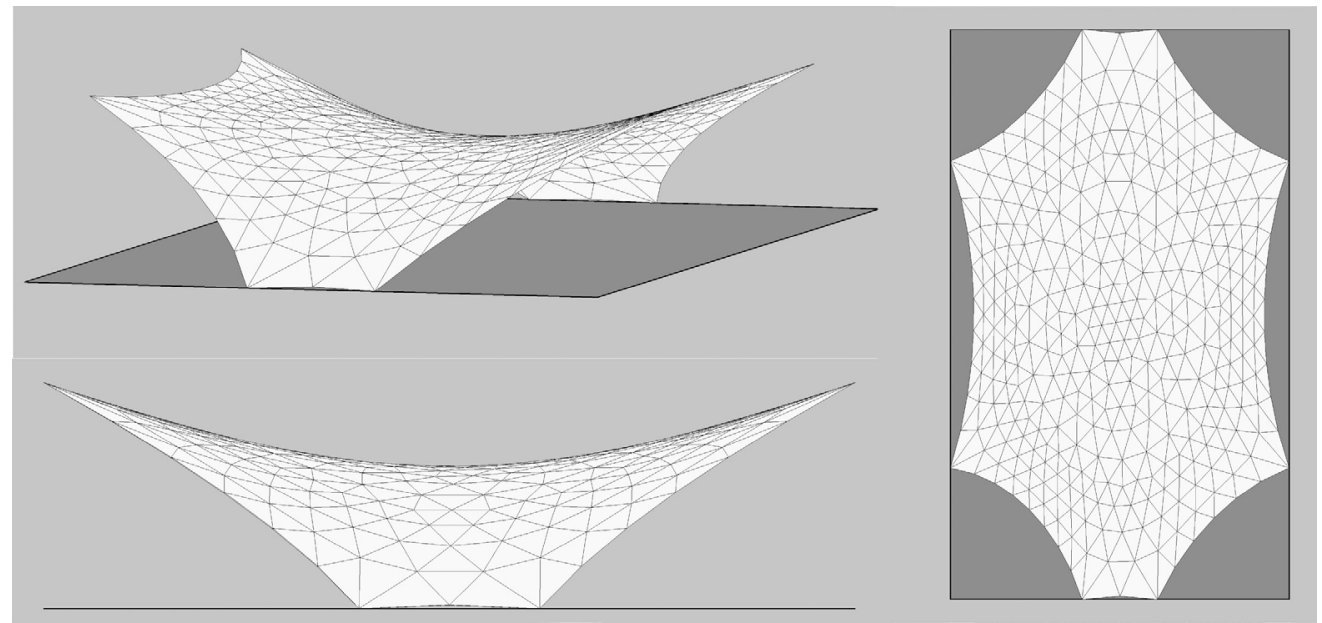

Figure 7. Initial equilibrium configuration with FDM and topological mapping methodology.

a)

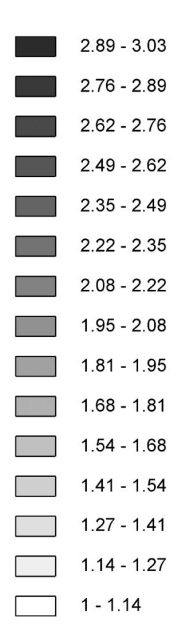

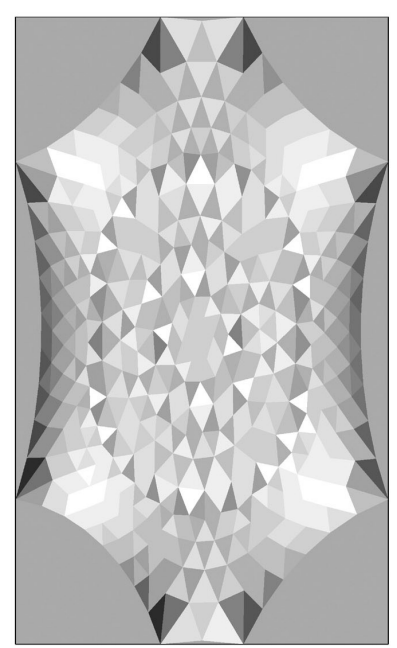

b)

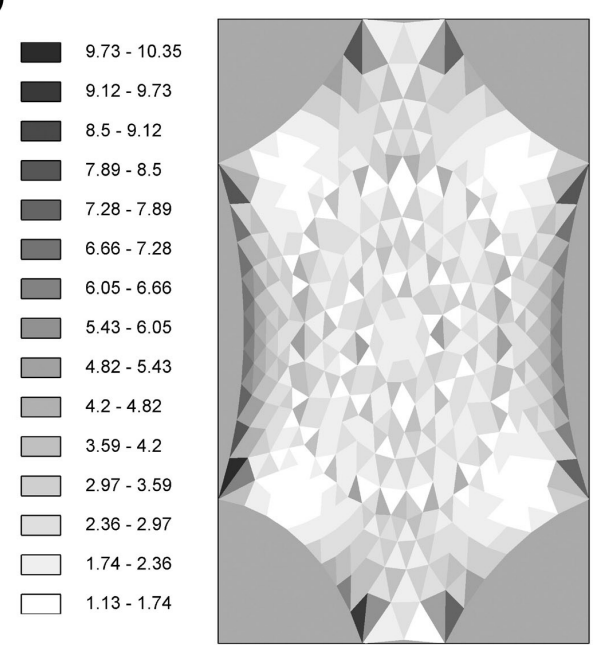

Figure 8. a) Major principal stress ( $\sigma 1)$, imposing 1 for the smaller value b) Ratio of principal stresses. 
Table 2. Error obtained in the iteration process.

\begin{tabular}{|c|c|c|c|c|c|c|c|c|c|c|}
\hline & \multicolumn{10}{|c|}{ Times through step $7=0$} \\
\hline ITER & $\mathbf{1}$ & 2 & 3 & 4 & 5 & 6 & 7 & 8 & 9 & 10 \\
\hline Error1 & $1,15 \mathrm{E}+01$ & $3,16 \mathrm{E}+00$ & $4,47 \mathrm{E}-01$ & $1,38 \mathrm{E}-02$ & $2,18 \mathrm{E}-\mathrm{O} 3$ & $5,58 \mathrm{E}-\mathrm{O} 4$ & $1,55 \mathrm{E}-\mathrm{O} 4$ & $5,61 \mathrm{E}-\mathrm{O} 5$ & $1,84 \mathrm{E}-\mathrm{O} 5$ & $6,76 \mathrm{E}-\mathrm{o} 6$ \\
\hline Error2 & \multicolumn{10}{|c|}{$1,26 \mathrm{E}+00$} \\
\hline & \multicolumn{10}{|c|}{ Times through step $7=1$} \\
\hline ITER & $\mathbf{1}$ & 2 & 3 & 4 & 5 & 6 & 7 & 8 & 9 & 10 \\
\hline Error1 & $1,26 \mathrm{E}+\mathrm{Oo}$ & $1,63 \mathrm{E}-02$ & 4,01E-04 & $7,83 \mathrm{E}-06$ & - & - & - & - & - & - \\
\hline Error2 & \multicolumn{10}{|c|}{$2,17 \mathrm{E}-01$} \\
\hline & \multicolumn{10}{|c|}{ Times through step $7=2$} \\
\hline ITER & $\mathbf{1}$ & 2 & 3 & 4 & 5 & 6 & 7 & 8 & 9 & 10 \\
\hline Error1 & $2,17 \mathrm{E}-\mathrm{O} 1$ & $2,86 \mathrm{E}-\mathrm{O} 3$ & $4,65 \mathrm{E}-06$ & - & - & - & - & - & - & - \\
\hline Error2 & \multicolumn{10}{|c|}{$8,71 \mathrm{E}-\mathrm{O} 2$} \\
\hline & \multicolumn{10}{|c|}{ Times through step $7=\mathbf{3}$} \\
\hline ITER & 1 & 2 & 3 & 4 & 5 & 6 & 7 & 8 & 9 & 10 \\
\hline Error1 & $8,71 \mathrm{E}-02$ & 9,67E-04 & 1,01E-06 & - & - & - & - & - & - & - \\
\hline Error2 & \multicolumn{10}{|c|}{$7,34 \mathrm{E}-02$} \\
\hline & \multicolumn{10}{|c|}{ Times through step $7=4$} \\
\hline ITER & $\mathbf{1}$ & 2 & 3 & 4 & 5 & 6 & 7 & 8 & 9 & 10 \\
\hline Error1 & $7,35 \mathrm{E}-02$ & $6,24 \mathrm{E}-04$ & $7,78 \mathrm{E}-07$ & - & - & - & - & - & - & - \\
\hline Error2 & \multicolumn{10}{|c|}{$6,89 \mathrm{E}-\mathrm{O} 2$} \\
\hline
\end{tabular}
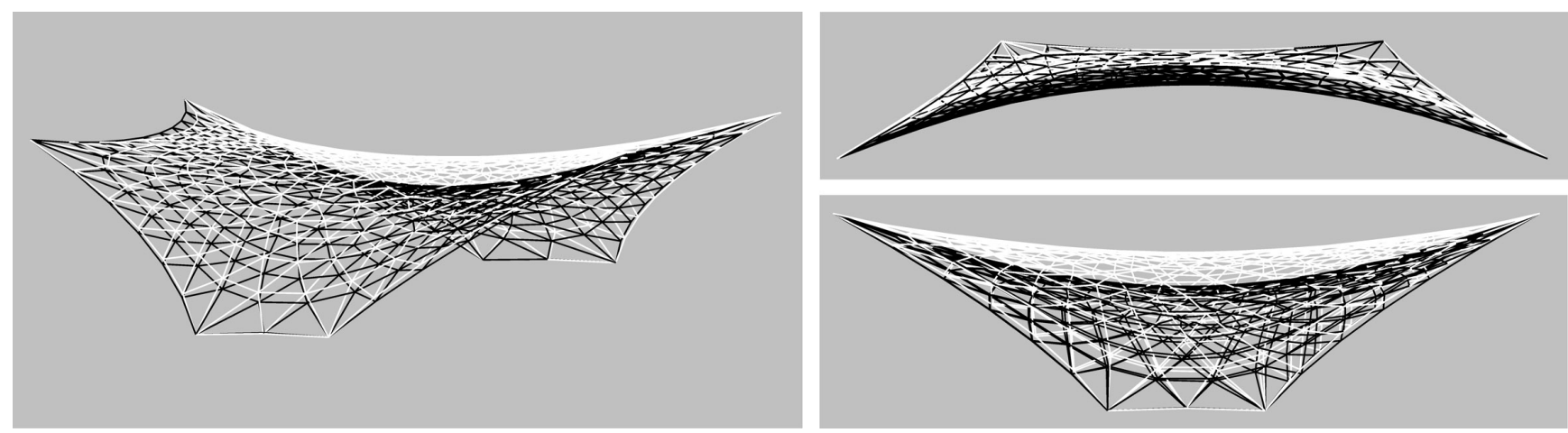

Figure 9. Initial and final configuration of the equilibrium shapes.

is obtained. In order to fulfill with the requirements of error Type 2, the numerical procedure has gone four times through step 7. Table 2 shows the resulting error for each step. Two types of error are computed: Error1 (Error Type 1) and Error2 (Error Type 2). It can be checked that after few iterations the resulting residual error for the final equilibrium configuration with the desired stresses is insignificant.

Figure 9 shows the initial configuration, obtained by the TMFDM, in black color, and the final configuration, with the specified stress field, in white color. A substantial change in the geometry can be observed.

\section{CONCLUSION}

An extended application of topological mapping to obtain a final equilibrium configuration of a membrane structure under controlled stress conditions is presented. The procedure deals with a topology based pin-joint network that is easy to implement and the force density method (FDM). The procedure is versatile and it represents a simple and straightforward method to achieve optimal equilibrium configurations through the use of finite element analysis tools for both refinements of the solutions and for their analysis under loading conditions.

\section{REFERENCES}

(1) Viglialoro, G., Murcia, J., Martinez, F. (2009). Equilibrium problems in membrane structures with rigid boundaries. Informes de la Construcción, 61(516):57-66, doi: http://dx.doi.org/10.3989/ic.08.038.

(2) Viglialoro, G., Murcia, J., Martinez, F. (2013). The 2-D continuous analysis versus the Density Force Method (discrete) for structural membrane equilibrium. Informes de la Construcción, 65(531): 349-358, doi: http://dx.doi.org/10.3989/ ic.11.086.

(3) Murcia, J. (2007). Structural membrane technology for footbridges. Informes de la Construcción, 59(507): 21-31, doi: http://dx.doi.org/10.3989/ic.2007.v59.i507.529. 
(4) Siev, A., Eidelman, J. (1964). Stress. Analysis of Prestressed Suspended Roofs. Proceedings ASCE, Journal of the Structural Division, ST4: 103-121.

(5) Schek, H.J. (1974). The force density method for form finding and computation of general networks. Computer Methods in Applied Mechanics and Engineering, 3(1): 115-134, doi: http://dx.doi.org/10.1016/0045-7825(74)90045-0.

(6) Haber, R.B., Abel, J.F. (1982). Initial equilibrium solution method for cable reinforced membranes. Part I: Formulations. Computer Methods in Applied Mechanics and Engineering, 30(3): 263-284, doi: http://dx.doi.org/10.1016/oo457825(82)90080-9.

(7) Barnes, M.R. (1988). Form-finding and analysis of prestressed nets and membranes. Computers \& Structures, 30(3): 685695, doi: http://dx.doi.org/10.1016/0045-7949(88)90304-5.

(8) Hernández-Montes, E., Jurado-Piña, R., Bayo, E. (2006). Topological mapping for tension structures. ASCE Journal of Structural Engineering, 132(6): 970-977, doi: http://dx.doi.org/10.1061/(ASCE)0733-9445(2006)132:6(970).

(9) Levy, R., Spillers, W.R. (2004). Analysis of Geometrically Nonlinear Structures, (2nd Ed.). London: Chapman \& Hall. 